\title{
Interactive comment on "Technical Note: A new approach to discriminate different black carbon sources by utilising fullerenes and metals in Positive Matrix Factorisation analysis of High-Resolution Soot Particle Aerosol Mass Spectrometer data" by Zainab Bibi et al.
}

\section{Anonymous Referee \#2}

Received and published: 6 December 2020

General comments:

This work attempts to integrate fullerene signals detected by the high-resolution sootparticle aerosol mass spectrometer (HR-SP-AMS) for source apportionment of ambient black carbon (BC) in an urban environment during the period with the bonfire event. The proposed positive matrix factorization (PMF) analysis has great potential to advance our understanding on the sources of $\mathrm{OA}$ and $\mathrm{BC}$ emitted from different 
combustion processes that cannot be easily resolved from conventional HR-AMS measurements.

As a technical note of ACP, my major concern is that this work did not demonstrate clearly how the additional fullerene signals can improve (1) the fundamental understanding (e.g., sources, transport, chemistry, etc.) in their case study and/or (2) black carbon source apportionment as the total fullerene signals are weak in general. These possibly can be achieved by comparing different PMF scenarios (e.g., including BC fragments in PMF with vs. without fullerene signals to see if different mass spectral profiles or number of factors may be obtained) if the results are available. I also have a few suggestion to improve the presentation quality of this work. Overall, the above general comments should be addressed together with the specific comments below in the revised version before considering to be published in ACP.

Major comments:

1. Introduction: Although the proposed method includes a new concept for data analysis, this manuscript should better recognize the contribution of other recent SP-AMS studies that performed fullerene detection near sources and that integrated $\mathrm{BC}$ signals in PMF for $\mathrm{BC}$ and $\mathrm{OA}$ source analysis.

2. Instrumentation: HR-SP-AMS has been deployed in many field studies with different configurations and operation modes. I do believe the tungsten vaporizer was removed from the instrument in this work as only BC-containing particles were detected. It would be very beneficial to readers who are not familiar with this instrument if the authors can explicitly describe whether the HR-SP-AMS was operated in the presence or absence of tungsten vaporizer and what can be detected with this specific configuration.

3. Lines 126-127: As there were only limited work to report metal detection in ambient particles using HR-SP-AMS, It is recommended to include a list of metal peaks that have been investigated and/or detected in this work.

Interactive

comment

Printer-friendly version

Discussion paper 
4. Lines 131-134: Please define fullerene peaks. If UMR data is used for large $\mathrm{m} / \mathrm{z}$, what is the possible error for determining fullerene signals?

5. Lines 137-138. There are a few previous field studies that included BC fragments for source apportionment/identification analysis of ambient BC and OA but they may not explicitly highlight this application in the manuscript. However, those publications should be cited here.

6. Figures: Although Figure 4 is good for visualization, it is recommended to report the Pearson correlation coefficients between different BC measurements here. This comment also applies to other time series comparisons throughout the manuscript. Furthermore, Figures 2-5 can be combined into a single graph with different panels so that the time series of different species can be easily compared. Figures 6 and 7 can be combined as well (i.e., showing HR-MS for lower range $\mathrm{m} / \mathrm{z}$ in Figure 6).

7. Section 3.5: Re-organization of this section is required. In particular, it is recommended to discuss the PMF factor profile and time series together instead of separating them into two sub-section as both of them provide information for sources of $B C$ and OA. For example, Figure 8 (time series of PMF factors) is required at the beginning of Section 3.5 when describing which OA factors were strongly associated with the bonfire night or other emissions. The mass spectral profile alone did not provide sufficient evidence to support the scientific argument.

8. Line234: The meaning of HULIS here is unclear. Figure 6 only shows "BC and HULIS" factor. The terminology throughout the manuscript should be consistent.

9. Lines 243-245: Both factors 2 and 3 consist of fullerene peaks. Please further elaborate how the fullerenes help to differentiate domestic burning and biomass burning during the bonfire event (e.g., any distinct peaks or mass spectral pattern that can be used?). It seems that the lower $\mathrm{m} / \mathrm{z}$ fragments are more than sufficient to tell the differences between the two OA factors. What does "hydrocarbon like fullerene" mean?

Printer-friendly version

Discussion paper
Interactive

comment

\section{Discussion paper}


10. Lines 287-288: Three bonfire night factors were identified. Were they all from bonfire emissions? If so, it implies that there were different types of bonfire emissions that can provide sufficient temporal variabilities for PMF factor separation. I am wondering if the same number of PMF factors can be obtained if fullerene signals is excluded. More discussion is required to demonstrate the importance of including fullerene signals in PMF analysis.

11. HULS factor: The manuscript mention a couple of times that a factor having strong $\mathrm{m} / \mathrm{z} 44$ signals can represent HULIS in ambient particles, but I cannot fully follow the flow of argument. My interpretation is that the mass spectral features of the HULIS factor is similar to that of more-oxidized oxygenated OA (MO-OOA) factor identified in most other field studies. I am wondering whether other co-located measurements in this work can provide evidence that the HULIS factor has some specific chemical features that cannot be described as MO-OOA. I understand this can be just a terminology issue. More elaboration is required here.

Minor comments: 1. Line 280. I think $\mathrm{m} / \mathrm{z} 73$ instead of $\mathrm{m} / \mathrm{z} 71$ for typical biomass burning factors. 2. Line 337: Please define BCtr. 3. Line 345: Please define BCwb.

Interactive comment on Atmos. Chem. Phys. Discuss., https://doi.org/10.5194/acp-2020-890, 2020. 